\title{
Postoperative excessive external femoral rotation in revision total hip arthroplasty is associated with muscle weakness in iliopsoas and gluteus medius and risk for hip dislocation
}

Hyonmin Choe ${ }^{1 *} \mathbb{D}$, Naomi Kobayashi², Daigo Kobayashi ${ }^{1}$, Shintaro Watanabe ${ }^{1}$, Koki Abe ${ }^{1}$, Taro Tezuka', Yusuke Kawabata', Masanobu Takeyama' and Yutaka Inaba'

\begin{abstract}
Background: Excessive external femoral rotation (FR) can functionally increase stem anteversion (SA) and is often observed at an early stage after surgery in revision total hip arthroplasty (THA). This study was conducted to investigate the prevalence of external FR, identify the factors associated with external FR, and determine the association of FR and other factors with hip dislocation in revision THA.

Methods: We enrolled 51 revision THA patients (55 hip cases). The patient background, angle of anatomical and functional SA, FR angle, sizes and densities of muscles around the hip joint, impingement distance, and consequence of postoperative hip dislocation were assessed by reviewing their medical history and imaging data that includes computed tomography (CT) scans before and after surgery.

Results: Forty-five hip cases (81.8\%) showed external FR (mean 13.0\%). External FR was significantly correlated with anatomical SA ( $r=-0.54)$ and increase in functional SA $(r=0.36)$, which was significantly correlated with impingement distance $(r=0.46)$. The independent factors associated with external FR in multivariate analysis were the anatomical SA, CT densities of the psoas, gluteus medius and maximus muscles, and 2-stage revision $\left(R^{2}=0.559\right)$. During follow-up period, eight cases of revision THA showed hip dislocation. FR, functional SA, impingement distance, CT density of psoas and gluteus medius muscle, body mass index, number of past operation, and ratio of 2-stage revision THA were significantly different between cases with dislocation and non-dislocation. The odds ratio of FR and impingement distance for hip dislocation was identified as 1.061 (95\% confidence interval (Cl): 1.011-1.114) and 0.901 (95\% Cl 0.820-0.991), respectively.
\end{abstract}

Conclusions: Revision THA frequently causes an external FR that functionally increases the SA and impingement risk, particularly in hips with 2-stage revision with psoas and gluteus medius muscle atrophy. Patients who have undergone revision THA and have an excessive external FR may require careful monitoring for possible hip dislocation due to hip joint instability and impingement.

Keywords: Revision total hip arthroplasty, Femoral rotation, Functional stem anteversion, Muscle atrophy, Impingement, Dislocation

\footnotetext{
*Correspondence: hyonmin@hotmail.com

1 Department of Orthopaedic Surgery, Yokohama City University, 3-9

Fukuura, Kanazawa-ku, Yokohama City, Kanagawa 236-0004, Japan

Full list of author information is available at the end of the article
} original author(s) and the source, provide a link to the Creative Commons licence, and indicate if changes were made. The images or other third party material in this article are included in the article's Creative Commons licence, unless indicated otherwise in a credit line to the material. If material is not included in the article's Creative Commons licence and your intended use is not permitted by statutory regulation or exceeds the permitted use, you will need to obtain permission directly from the copyright holder. To view a copy of this licence, visit http://creativecommons.org/licenses/by/4.0/. The Creative Commons Public Domain Dedication waiver (http://creativecommons.org/publicdomain/zero/1.0/) applies to the data made available in this article, unless otherwise stated in a credit line to the data. 


\section{Background}

Using the combined anteversion technique for the cup and stem anteversion (SA) angle can minimize the risk of hip impingement during daily hip motions in total hip arthroplasty (THA) or revision THA patients [1-4]. Recent studies demonstrated the important effects of pelvic and femoral positions on anatomical and functional changes in the cup and SA angles, revealing the importance of tailored planning for the cup and SA that depends on the posture of THA patients [5-7]. Recurrent dislocation is among the major complications of THA, and the incidence rate of dislocation is higher in revision THA patients than in primary THA patients $[8,9]$. In revision cases, excessive external rotation of the femur is often observed at an early stage after surgery, although several studies demonstrated that internal changes occur in the femoral position of primary THA patients [4, 7]. An external femoral rotation (FR) in revision THA patients may increase the functional angle of SA or risk of prosthetic or bony impingement and risk of hip dislocation in leg extension position, but this relationship has not yet been investigated.

The muscles around the hip joint affect its stability, which is an important factor in preventing postoperative hip dislocation in THA patients [10-12]. The muscles may also affect the FR alignment. Hence, muscle atrophy due to surgical damage or disuse may have a greater effect on the postoperative femoral position in revision THA patients than in primary THA patients. Previous studies focused on the effect of abductor muscles on hip dislocation [10, 13-15]; however, the association of muscles around the hip joint (including the psoas, iliac, and gluteus maximus (Gmax) and medius (Gmed)) with the FR angle or hip dislocation remains unclear.

Therefore, this study was conducted to investigate the prevalence of external FR in revision THA patients, identify the factors associated with external FR, and determine the association of FR and the other factors with the risk of hip dislocation in revision THA.

\section{Methods}

\section{Patient analysis}

This retrospective study was approved by the institutional ethics review board of Yokohama City University (B200100008). The records of 123 patients who underwent revision THA in Yokohama City University Hospital from 2006 to 2019 were reviewed. The exclusion criteria were as follows: replacement of the head or liner only, uncontrollable periprosthetic joint infection (PJI), did not provide consent to participate, no follow-up after 6 months, and failure to undergo X-ray and computed tomography $(\mathrm{CT})$ scans both before and after revision

\section{3 patients with revision THA}

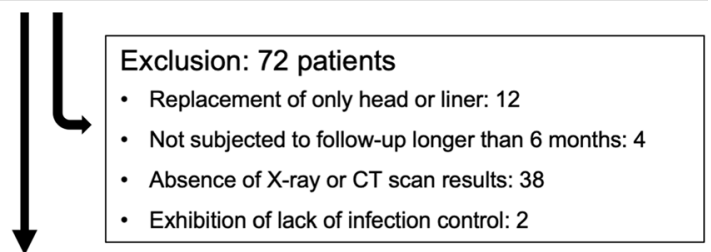

\section{1 patients (55 hips) with revision THA}

- 1-stage revision THA: 33 hips

- 2-stage revision THA: 22 hips

Fig. 1 Flowchart of revision total hip arthroplasty (THA) patient enrolment

Table 1 Demographic, surgical, and CT data in the revision THA cohort

\begin{tabular}{ll} 
Demographic data & \\
Age (years) & $70.2(9.6)$ \\
Female ratio (\%) & 73 \\
Body mass index $\left(\mathrm{kg} / \mathrm{m}^{2}\right)$ & $23.4(4.8)$ \\
Diagnosis (hips) & Aseptic: 32, septic: 23 \\
Preoperative femoral rotation & $11.3(19.1)$ \\
Surgical data & \\
Surgical procedure (hips) & 1 -stage: 33; 2-stage: 22 \\
Leg length extension (mm) & $11.9(18.3)$ \\
Change of femoral offset (mm) & $-0.1(9.5)$ \\
Postoperative anatomical stem anteversion & $24.7(14.7)$ \\
Preoperative muscle sizes (mm²) & \\
Psoas muscle & $500(31.8)$ \\
Iliac muscle & $555(39)$ \\
Gluteus maximus & $1926(660)$ \\
Gluteus medius & $1688(849)$ \\
Preoperative CT density of muscles & \\
Psoas muscle (HU) & $35.2(3.3)$ \\
Iliac muscle (HU) & $55.3(46.0)$ \\
Gluteus maximus (HU) & $4.2(2.9)$ \\
Gluteus medius (HU) & $12.1(35.3)$ \\
\hline
\end{tabular}

Data represent the mean values (standard deviation)

HU, Hounsfield unit

THA (Fig. 1). Our study cohort included 55 hip cases in 51 patients (37 women and 14 men) with a mean age of 70 years and mean body mass index (BMI) of $23.4\left(\mathrm{~kg} / \mathrm{m}^{2}\right)$ (Table 1). All study participants underwent a hip X-ray in the frontal view and CT scan (Sensation 16; Siemens AG, Erlangen, Germany; $120 \mathrm{kV}$ and $300 \mathrm{~mA}, 1.5 \mathrm{~mm}$ slice) from the first lumbar spine to the lower edge of femur 
before and within a month of surgery using a calibration phantom (B-MAS 200; Kyoto-Kagaku, Japan) and dedicated software (SYNAPSE Enterprise-PACS, Fujifilm, Tokyo, Japan). Muscle CT densities, measured in Hounsfield units, were adjusted based on the reference values from the calibration phantom [16].

In the 55 revision THA cases, 23 hip cases were diagnosed with PJI using the modified Musculoskeletal Infection Society criteria [17] and 32 hip cases were diagnosed with aseptic loosening, including one pseudotumor. All 32 aseptic loosening patients underwent 1-stage revision surgery. Among the 23 PJI patients, one patient underwent 1 -stage revision surgery and 22 patients underwent 2-stage THA using a hydroxyapatite block containing an antimicrobial (Bone Ceram P, Olympus Terumo Biomaterials Corp, Tokyo, Japan) (Table1) [18]. During the study period, 8 hips in 8 patients experienced postoperative dislocation, and all 8 patients were enrolled in the current study. Among these patients, 6 cases were conservatively treated and 2 cases were subjected for re-revision THA.

Patient characteristics were investigated. The leg and femoral offset extension levels before and after surgery were investigated by performing a frontal view hip X-ray.
FR alignments were assessed by measuring the anatomical and functional SA angle and FR angle using crosssectional CT imaging before and after surgery (Fig. 2A) $[4,7]$. The shortest posterior pelvis-stem distance was measured as the prosthetic impingement distance, and the shortest posterior pelvis-femur distance was measured as the bony impingement distance (Fig. 2A) using cross-sectional CT imaging after surgery. The shortest distance between the pelvis-stem and pelvis-femur was defined as the impingement distance. As a representative measure of muscle quality, preoperative cross-sectional CT images were used to evaluate the sizes and densities of the psoas, iliac, Gmax, and Gmed muscles using the calibration phantom (Fig. 2B) [16]. The prevalence of external FR in revision THA patients, factors associated with external FR, and association of these factors with the risk of hip dislocation in revision THA were investigated.

\section{Statistical analysis}

Statistical significance was determined using Student's $t$ test or nonparametric Mann-Whitney U test. Correlations were analyzed using Pearson's correlation analysis. A forward-backward selection method was used for
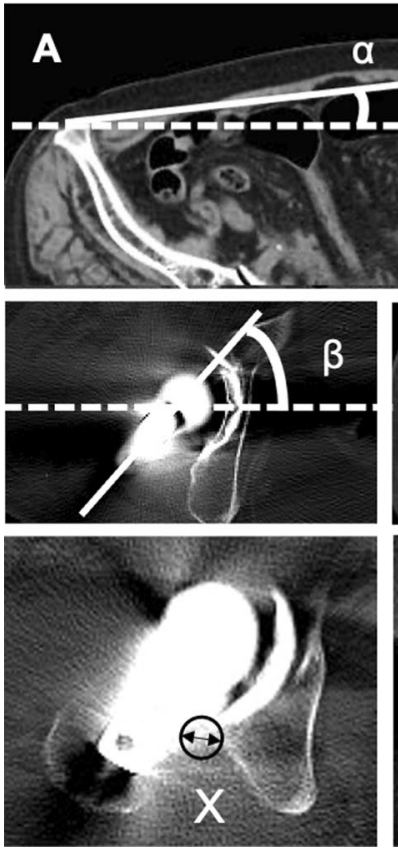

Fig. 2 Computed tomography (CT) measurements of femoral rotation angle, impingement distance, and muscle size and density. A Functional stem anteversion or external rotation angle of the femur was measured by subtracting the value for the rotation angle of the superior anterior iliac spine line from that of the stem angle ( $\beta-a)$ or angle of the posterior femoral condyle axis $(\gamma-a)$ using a CT image acquired within a month of surgery. The posterior pelvis-stem or pelvis-femur distances were measured by drawing a circle and by measuring the shortest distance from the stem to the pelvis $(X)$ or from the femur to pelvis $(Y)$ at the lower level of the artificial head in a cross-sectional $C T$ image acquired after surgery. B Using preoperative CT images, the psoas muscle was detected at the upper edge of the iliac crest, and the iliac, gluteus medius, and gluteus maximus muscles were detected at the level of the mid-point between the iliac crest and great trochanter. A calibration phantom was placed during the CT scan and utilized to adjust the CT density, which was measured in Hounsfield units. (Gmed, gluteus medius; Gmax, gluteus maximus) 
multiple regression analysis. The odds ratio for dislocation was calculated by logistic regression analysis. All statistical analyses were conducted using JMP ${ }^{\circledR} 15$ software (SAS Institute, Inc., Cary, NC, USA). P values $<0.05$ were considered to indicate statistically significant results. All graphs were prepared using GraphPad Prism 8 software (GraphPad, Inc., CA, USA).

\section{Results}

Among the 51 patients with 55 hips who underwent revision THA, 45 hip cases $(81.8 \%)$ had external FR with a mean angle of $13.0^{\circ}$ [standard deviation $\pm 12.6^{\circ}$ ] postoperatively, including 14 hip cases $(25.5 \%)$ with a more than $30^{\circ}$ external FR with a maximum of $56^{\circ}$ in revision THA (Fig. 3A).

The external FR increased the SA from anatomical to functional (from mean $24.7^{\circ}$ to $36.7^{\circ} ; P=0.01$ ). The significant correlation between the external rotation of the femur and functional SA $(r=0.36)$ indicated the importance of the FR angle on functional SA (Fig. 3B). Both external FR and the functional SA showed a significantly negative correlation with an impingement distance in the leg extension position ( $r=0.30,0.46$, respectively; Fig. $3 \mathrm{C}$ and $3 \mathrm{D}$ ). As a surgical factor, 2-stage revision THA showed a significantly higher external FR than 1-stage revision THA (mean $17.2 \pm 12.7^{\circ}$ vs. $10.1 \pm 12.0^{\circ}, P=0.03$; Fig. $4 \mathrm{~A}$ ).

Among the patient factors, the CT densities of psoas, iliac, and Gmed muscles and presence of infection were significantly associated with an increased external FR angle (Table 2; Figs. 4B and 4C). In contrast, the postoperative anatomical SA was significantly negatively correlated with external FR (Fig. 4D). In multivariate analysis, postoperative anatomical SA; CT densities of the psoas, Gmax, and Gmed muscles; and 2-stage revision were identified as independent factors associated with an external FR with the following regression equation $\left(R^{2}=0.559\right)$ (Table 3$)$ :

Postoperative FR angle $=29.5-0.399 \times$ postoperative anatomical stem anteversion -0.129

$\times$ CT density of psoas $+0.129 \times$ CT density of Gmax -0.172

$\times$ CT density of Gmed $-3.291 \times$ revision surgery (1 stage: 0,2 stage: 1$)$
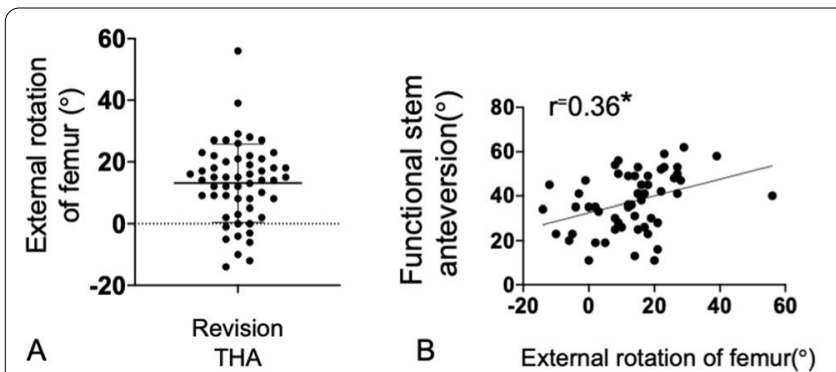

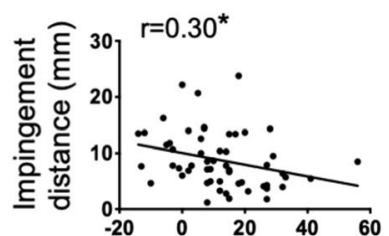

External rotation of femur $\left({ }^{\circ}\right)$

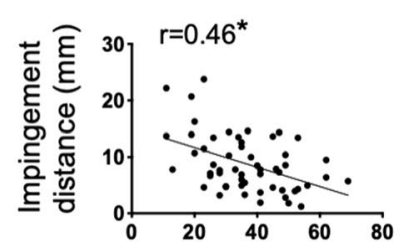

Functional stem anteversion $\left({ }^{\circ}\right)$

Fig. 3 Femoral rotation (FR) angle and stem anteversion (SA) in revision and impingement distance. A External FR angle mean of $13.0^{\circ}$ up to $56^{\circ}$. Among the 55 revision THA cases, 45 cases showed an external rotation, and 14 showed an external rotation of more than $30^{\circ}$. $\mathbf{B}$ External FR is correlated with functional SA in both revision and primary THA patients ( $r=0.36, P<0.01$; Pearson's correlation analysis). C, D External FR or functional SA was correlated with the impingement distance in revision THA patients $(r=0.30,0.46$, respectively; $P<0.01$; Pearson's correlation analysis)
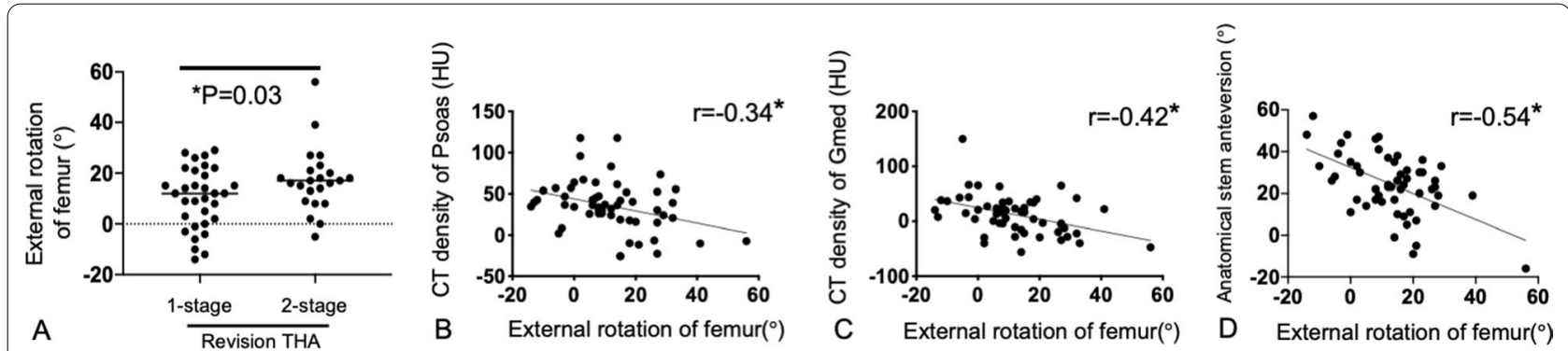

Fig. 4 Patient and surgical factors associated with external rotation of the femur. A 2-stage revision total hip arthroplasty had higher external FR than 1-stage revision total hip arthroplasty (mean $17.2 \pm 12.7^{\circ} \mathrm{vs} .10 .1 \pm 12.0^{\circ} ; P=0.03$ ). B-D CT densities for the psoas and gluteus medius muscles, and anatomical stem anteversion were negatively correlated with the external rotation of the femur $(R=0.34,0.42$, and 0.54 , respectively; $P<0.01$; Pearson's correlation analysis) 
Table 2 Factors correlated with femoral rotation

\begin{tabular}{lcl}
\hline Person's correlation & $\boldsymbol{r}$ & $\boldsymbol{P}$ value \\
\hline Age (years) & -0.01 & 0.93 \\
Body mass index $\left(\mathrm{kg} / \mathrm{m}^{2}\right)$ & 0.10 & 0.740 \\
Preoperative femoral rotation & 0.11 & 0.44 \\
Leg length extension & -0.26 & 0.06 \\
Change of femoral offset & -0.14 & 0.29 \\
Postoperative anatomical stem anteversion & -0.54 & $0.001^{*}$ \\
Size of psoas muscle & -0.09 & 0.53 \\
Size of iliac muscle & -0.17 & 0.22 \\
Size of gluteus maximus & -0.05 & 0.71 \\
Size of gluteus medius & -0.09 & 0.49 \\
CT density of psoas muscle & -0.29 & $0.03^{*}$ \\
CT density of iliac muscle & -0.31 & $0.03^{*}$ \\
CT density of gluteus maximus & -0.17 & 0.22 \\
CT density of gluteus medius & -0.39 & $0.004^{*}$ \\
\hline
\end{tabular}

Postoperative hip dislocation occurred in 8 hips during the follow-up period. Although the anatomical SA was not significantly different between revision THA patients with dislocation and non-dislocation, external FR and functional SA were significantly high in patients with dislocation (Table 4). The other factors of impingement distance, CT density of psoas, Gmed, and Gmax, body mass index, number of past operation, and ratio of 2-stage revision THA were also significantly different between patients with dislocation and non-dislocation (Table 4). Increased external FR, low impingement distance, low CT density of the psoas, Gmed muscle, BMI, 2-stage revision THA, and numbers of past operations were identified as significant risk factors for hip dislocation (Table 5).

Table 3 Multiple regression analysis for predicting femoral rotation after surgery in revision total hip arthroplasty patients

\begin{tabular}{llll}
\hline Variable & $\boldsymbol{B}$ & $\boldsymbol{\beta}$ & $\boldsymbol{P}$-value \\
\hline Constant & 29.5 & -0.26 & 0.000 \\
2 stage revision & -3.291 & -0.46 & 0.01 \\
Postoperative & -0.399 & -0.31 \\
Anatomical stem anteversion & -0.129 & 0.26 \\
CT density of psoas muscle & 0.129 & -0.48 \\
CT density of gluteus maximus (Gmax) & -0.172 & 0.000 \\
CT density of gluteus medius (Gmed) & $29.5-0.399 \times$ postoperative anatomical stem anteversion \\
Regression formula for angle of postoperative femoral & $-0.129 \times C$ density of psoas $+0.129 \times C$ density of Gmax \\
rotation & $-0.172 \times C T$ density of Gmed $(-3.291$ with 2 stage revision) \\
& $\left(R^{2}=0.559\right)$ & 0.03 \\
\end{tabular}

Table 4 Comparison of factors between dislocation $(n=8)$ and non-dislocation $(n=47)$

\begin{tabular}{|c|c|c|}
\hline Factors & Data & $P$ value \\
\hline Comparison of ratio for dislocation (\%) & & Chi-square test \\
\hline Diagnosis & PJl: 5/23(22\%) versus aseptic: 3/32(9\%) & $<0.01$ \\
\hline Surgical procedure & 2-stage: $5 / 22(23 \%)$ versus 1 -stage: $3 / 33(9 \%)$ & $<0.01$ \\
\hline Comparison of mean values [standard deviation] & & $t$ test \\
\hline $\mathrm{BMI}\left(\mathrm{kg} / \mathrm{cm}^{2}\right)$ & $27.2[1.5]$ versus $23.2[0.44]$ & $<0.01$ \\
\hline CT density of Psoas muscle (HU) & $13.3[25.1]$ versus $37.4[23.2]$ & $<0.01$ \\
\hline $\mathrm{CT}$ density of gluteus maximus (HU) & $-6.9[25.1]$ versus $13.5[22.1]$ & $<0.05$ \\
\hline CT density of gluteus medius (HU) & -6.9 [13.6] versus $24.5[28.6]$ & $<0.01$ \\
\hline External rotation of femur $\left(^{\circ}\right)$ & $18.5[9.1]$ versus $4.8[14.3]$ & $<0.01$ \\
\hline Functional stem anteversion $\left(^{\circ}\right)$ & $43.6[10.5]$ versus $34.5[12.5]$ & $<0.05$ \\
\hline Impingement distance(mm) & $13.7[9.7]$ versus $21.3[9.1]$ & $<0.05$ \\
\hline Numbers of past operation & $2.4[1.8]$ versus $1.5[0.9]$ & $<0.05$ \\
\hline
\end{tabular}


Table 5 Odds ratio for postoperative dislocation

\begin{tabular}{lllll}
\hline & Odd ratio & \multicolumn{2}{c}{$\mathbf{9 5 \% \text { confidence interval }}$} \\
\cline { 3 - 4 } & & Lower limit & Upper limit \\
\hline External rotation of femur & 1.061 & 1.011 & 1.114 \\
Impingement distance & 0.901 & 0.820 & 0.991 & 0.016 \\
CT density of psoas muscle & 0.957 & 0.926 & 0.988 & 0.031 \\
CT density of gluteus medius & 0.963 & 0.938 & 0.989 & 0.007 \\
BMI & 1.178 & 1.022 & 1.358 & 0.005 \\
2-stage revision THA & 5.268 & 1.620 & 17.131 & 0.024 \\
Numbers of past operation & 2.289 & 1.322 & 3.963 & 0.006 \\
\hline
\end{tabular}

\section{Discussion}

Operative planning to achieve an optimal combined cup and SA is important for successful THA [1-3]. Previous reports revealed that internal changes in the FR occur in primary THA patients $[7,19]$. As an excessive external femoral position is occasionally observed at an early stage after surgery in revision THA cases, we first focused on the FR angle and functional SA, as well as their relation to the posterior impingement distance in revision THA patients. In our study population, more than $80 \%$ of revision THA patients showed external FR that functionally increased the SA and posterior impingement risk in leg extension. Because the tendency of internal femoral changes in primary THA patients does not typically contribute to posterior impingement and the risk of hip dislocation, we investigated whether the external FR and impingement risk was a one cause of the higher dislocation rate in revision THA patients $[8,9,20]$. As a result, a higher external FR and lower impingement distance were identified as significant risk factor for postoperative hip dislocation in our cohort, indicating that revision THA patients with an excessive external FR position require careful assessment of hip dislocation.

Abductor deficiency contributes to hip instability [9, $10,13-15,20]$, indicating the necessity of quantifying the size and density of muscles around the hip joint and assessing the associations between them and the femoral rotational angle or hip dislocation. For this purpose, we utilized previously established methods to evaluate the muscle size and density via cross-sectional CT imaging, which can reflect muscle strength [16]. Interestingly, our CT analysis revealed a significant correlation between muscle weakness in the psoas, iliac, or Gmed muscles and external FR. This can be explained by the internal rotational function of these muscles, in contrast to the Gmax function during external rotation. Therefore, the greater increase in FR in patients with septic loosening who mostly underwent 2 -stage revision THA likely occurred because of the atrophic effect of the greater surgical intervention or longer disuse period on these muscles in 2-stage revision patients. Our CT analysis also revealed a negative correlation between the postoperative SA and FR, similar to the findings of a previous study of primary THA patients [7]. This paradoxical effect may also be explained by the fact that a higher SA induces higher tensioning of the iliopsoas or Gmed but lower tensioning of the Gmax [7].

Multiple regression analysis revealed the independent functions of the psoas and Gmed muscles on the internal FR. Nevertheless, the psoas and Gmed muscles reportedly contribute to hip stability $[9,10,13-$ 15]. The significant correlations of these muscles with FR indicate that the excessive external FR position in revision THA patients is an important clinical sign of muscle weakness in the psoas and Gmed muscles. As these muscles are well-known and important stabilizers of the hip joint $[9,10,13-15]$, we additionally investigated the association of these muscles with the hip dislocation risk. As a result, low CT densities of the psoas and medius muscle were also identified as risk factors for hip dislocation. Therefore, patients with an excessive external FR may have the risk factors of hip instability because of muscle atrophy and hip impingement due to an increased functional SA for postoperative hip dislocation.

Our study had several limitations. First, more than $50 \%$ of the revision THA patients treated at our hospital were not included in retrospective evaluations because of a lack of medical records, clinical followup, and image assessment. However, we included all dislocation cases during the study period that may have resulted in successful analysis of the association of FR with dislocation. Secondly, we did not evaluate mid- and long-term changes in FR in our study cases. However, our study is the first to demonstrate the tendency towards an external FR, and to reveal an association between the lower muscle CT density and early 
postoperative posture in a revision THA cohort. SA modification or muscle strengthening may be required in these patients; however, further studies are needed to validate this possibility.

In conclusion, more than $80 \%$ of revision THA caused an external FR that functionally increased the SA and impingement risk in leg extension, particularly in hips with 2-stage revision with psoas and Gmed muscle atrophy. Revision THA patients with an excessive external FR may require careful monitoring for hip dislocation due to instability and impingement.

\section{Abbreviations}

SA: Stem anteversion; THA: Total hip arthroplasty; FR: Femoral rotation; Gmax: Gluteus; Gmed: Medius; BMI: Body mass index.

\section{Acknowledgements}

None.

\section{Authors' contributions}

$\mathrm{HC}$ designed the experiments, conducted the experiments, and wrote the paper. NK contributed on the design of the experiments and wrote the paper. DK, SW, KA, TT, YK, and MT contributed on the conduction of experiments and analysis of data. YI contributed on design of the experiments and writing of paper.

\section{Funding}

This research did not receive any specific grant from funding agencies in the public, commercial, or not-for-profit sectors.

\section{Availability of data and materials}

The datasets used and/or analyzed during the current study are available from the corresponding author on reasonable request.

\section{Declarations}

\section{Ethics approval and consent to participate}

This retrospective study was approved by our institutional ethics review board (B200100008). Patients consent was obtained in the form of opt-out on the website.

\section{Consent for publication}

The author consents to publication of the work.

\section{Competing interests}

The authors declare that they have no competing interests.

\section{Author details}

1 Department of Orthopaedic Surgery, Yokohama City University, 3-9 Fukuura, Kanazawa-ku, Yokohama City, Kanagawa 236-0004, Japan. ${ }^{2}$ Department of Orthopaedic Surgery, Yokohama City University Medical Center, 4-57 Urahune-cho, Minami-ku, Yokohama City, Kanagawa 232-0024, Japan.

Received: 2 August 2021 Accepted: 21 September 2021

Published online: 09 October 2021

\section{References}

1. Widmer $\mathrm{KH}$, Zurfluh B. Compliant positioning of total hip components for optimal range of motion. J Orthop Res. 2004;22(4):815-21.
2. Nakashima Y, Hirata M, Akiyama M, Itokawa T, Yamamoto T, Motomura $\mathrm{G}$, et al. Combined anteversion technique reduced the dislocation in cementless total hip arthroplasty. Int Orthop. 2014;38(1):27-32.

3. Dorr LD, Malik A, Dastane M, Wan Z. Combined anteversion technique for total hip arthroplasty. Clin Orthop Relat Res. 2009;467(1):119-27.

4. Uemura K, Takao M, Otake Y, Koyama K, Yokota F, Hamada H, et al. Can anatomic measurements of stem anteversion angle be considered as the functional anteversion angle? J Arthroplast. 2018;33(2):595-600.

5. Ishida T, Inaba Y, Kobayashi N, Iwamoto N, Yukizawa Y, Choe H, et al. Changes in pelvic tilt following total hip arthroplasty. J Orthop Sci. 2011;16(6):682-8.

6. Suzuki H, Inaba Y, Kobayashi N, Ishida T, Ike H, Saito T. Postural and chronological change in pelvic tilt five years after total hip arthroplasty in patients with developmental dysplasia of the hip: a three-dimensional analysis. J Arthroplast. 2016;31(1):317-22.

7. Tezuka T, Inaba Y, Kobayashi N, Choe H, Higashihira S, Saito T. The influence of patient factors on femoral rotation after total hip arthroplasty. BMC Musculoskelet Disord. 2018:19(1):189.

8. Faldini C, Stefanini N, Fenga D, Neonakis EM, Perna F, Mazzotti A, et al. How to prevent dislocation after revision total hip arthroplasty: a systematic review of the risk factors and a focus on treatment options. J Orthop Traumatol. 2018;19(1):17

9. Huten D, Fournier Y, Gicquel T, Bertho P, Basselot F, Hamadouche M. Risk factors for dislocation after revision total hip arthroplasty with a dual-mobility cup. Matched case-control study (16 cases vs. 48 controls). Orthop Traumatol Surg Res. 2019;105(7):1303-9.

10. Yoshimoto K, Nakashima Y, Yamamoto T, Fukushi J-I, Motomura G, et al. Dislocation and its recurrence after revision total hip arthroplasty. Int Orthop. 2016:40(8):1625-30.

11. Brown TD, Elkins JM, Pedersen DR, Callaghan JJ. Impingement and dislocation in total hip arthroplasty: mechanisms and consequences. lowa Orthop J. 2014;34:1-15

12. Folinais $D$, Thelen $P$, Delin $C$, Radier C, Catonne Y, Lazennec JY. Measuring femoral and rotational alignment: EOS system versus computed tomography. Orthop Traumatol Surg Res. 2013;99(5):509-16.

13. Alberton GM, High WA, Morrey BF. Dislocation after revision total hip arthroplasty: an analysis of risk factors and treatment options. J Bone Joint Surg Am. 2002;84(10):1788-92.

14. Wetters NG, Murray TG, Moric M, Sporer SM, Paprosky WG, Della Valle CJ. Risk factors for dislocation after revision total hip arthroplasty. Clin Orthop Relat Res. 2013;471(2):410-6.

15. Yoshimoto K, Nakashima Y, Aota S, Kaneuji A, Fukui K, Hirakawa K, et al. Re-dislocation after revision total hip arthroplasty for recurrent dislocation: a multicentre study. Int Orthop. 2017;41(2):253-8.

16. Momose T, Inaba Y, Choe H, Kobayashi N, Tezuka T, Saito T. CT-based analysis of muscle volume and degeneration of gluteus medius in patients with unilateral hip osteoarthritis. BMC Musculoskelet Disord. 2017;18(1):457

17. Parvizi J, Gehrke T, International Consensus Group on Periprosthetic Joint I. Definition of periprosthetic joint infection. J Arthroplast. 2014;29(7):1331.

18. Choe H, Inaba Y, Kobayashi N, Miyamae Y, Ike H, Saito T. Clinical utility of antibiotic-loaded hydroxyapatite block for treatment of intractable periprosthetic joint infection and septic arthritis of the hip. Mod Rheumatol. 2015;25(6):937-42.

19. Kobayashi D, Choe H, Kobayashi N, Tezuka T, Ike H, Inaba Y. Association of femoral rotation with whole-body alignment in patients who underwent total hip arthroplasty. Arthroplast Today. 2020;6(3):532-7.

20. Perticarini L, Rossi SMP, Fioruzzi A, Jannelli E, Mosconi M, Benazzo F. Modular tapered conical revision stem in hip revision surgery: mid-term results. BMC Musculoskelet Disord. 2021;22(1):29.

\section{Publisher's Note}

Springer Nature remains neutral with regard to jurisdictional claims in published maps and institutional affiliations. 